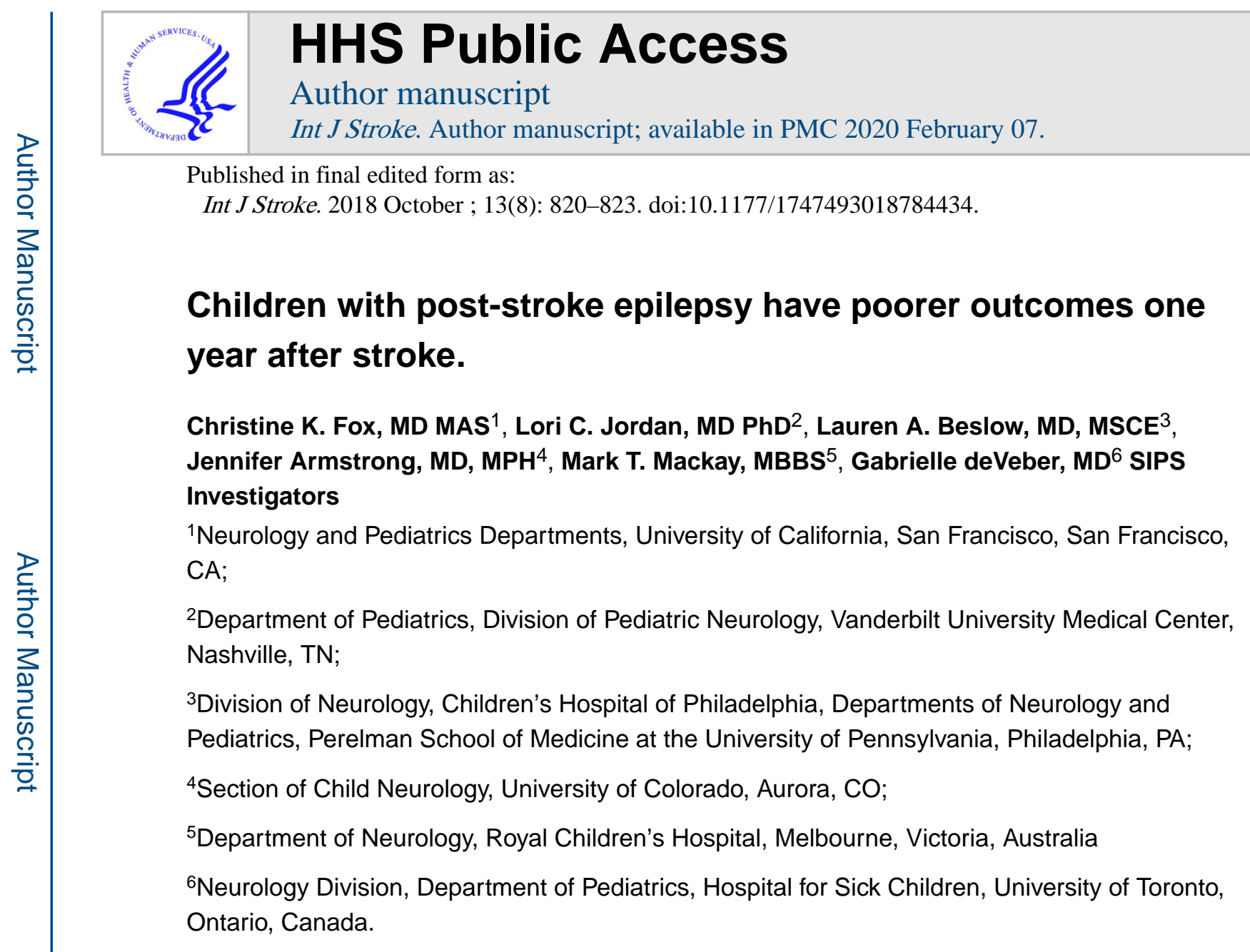

Published in final edited form as:

Int J Stroke. 2018 October ; 13(8): 820-823. doi:10.1177/1747493018784434.

\title{
Children with post-stroke epilepsy have poorer outcomes one year after stroke.
}

\author{
Christine K. Fox, MD MAS ${ }^{1}$, Lori C. Jordan, MD PhD², Lauren A. Beslow, MD, MSCE ${ }^{3}$, \\ Jennifer Armstrong, MD, MPH ${ }^{4}$, Mark T. Mackay, MBBS ${ }^{5}$, Gabrielle deVeber, MD ${ }^{6}$ SIPS \\ Neurology and Pediatrics Departments, University of California, San Francisco, San Francisco, \\ ${ }^{2}$ Department of Pediatrics, Division of Pediatric Neurology, Vanderbilt University Medical Center, \\ Nashville, TN; \\ ${ }^{3}$ Division of Neurology, Children's Hospital of Philadelphia, Departments of Neurology and \\ ${ }^{4}$ Section of Child Neurology, University of Colorado, Aurora, CO; \\ Ontario, Canada.
}

\begin{abstract}
Background: To measure the association of epilepsy and neurologic outcome after childhood arterial ischemic stroke.

Methods: Prospective cohort study of children (29 days -19 years) enrolled after acute arterial ischemic stroke at 21 international pediatric stroke centers and followed to identify epilepsy. One year post-stroke, outcomes were scored using the examination-based Pediatric Stroke Outcome Measure (PSOM, range 0-10); higher values reflect greater disability. Ordinal logistic regression was used to measure the association of PSOM scores (categorized as $0-1,1.5-3,3.5-6,6.5-10$ ) with epilepsy.
\end{abstract}

Results: Investigators enrolled 86 children [median age 6.1 years, interquartile range (IQR) 1.4 12. 2 years] with acute stroke. At one year, 18/80 (23\%) remained on an anti-convulsant including 8/80 (10\%) with epilepsy. Among the 70 with PSOM scored, the median was 0.5 (IQR 0-1.5) for children without epilepsy ( $\mathrm{n}=63$ ), and 6 (IQR 0.5-10) for children with epilepsy ( $\mathrm{n}=7$ ). In univariable analyses, poorer 1-year outcome was associated with middle cerebral artery stroke,

Correspondence: Christine Fox, MD MAS, 675 Nelson Rising Lane, 402B, San Francisco, CA 94134, Christine.Fox@ucsf.edu. Contributors:

Drs. Fox, Mackay and deVeber contributed to study design.

Dr. Fox performed statistical analyses and drafted the manuscript.

Drs. Fox, Jordan, Beslow, Armstrong, Mackay and deVeber substantially contributed to the acquisition and interpretation of data; critically reviewed and revised it for important intellectual content, approved the final work and have participated sufficiently to take responsibility for the content.

The authors declare no conflicts of interest. 
cortical infarcts, hemorrhagic transformation, longer acute seizure duration, hospital disposition not to home, and epilepsy. In multivariable analysis, middle cerebral artery stroke [odds ratio (OR) 4.9, 95\% confidence intervals (CI) 1.1-21.3] and epilepsy (OR 24.1, CI 1.5-380) remained associated with poorer outcome.

Conclusions: Children who developed epilepsy during the first year post-stroke had poorer neurologic outcomes than those without epilepsy.

\section{Keywords}

Ischemic stroke; Risk factors; Seizures; Pediatric; Outcomes; Neurology

\section{Introduction.}

Epilepsy is a common complication of pediatric stroke, with a 10-year cumulative incidence of approximately $30 \%$ among childhood stroke survivors. ${ }^{1}$ Children with post-stroke epilepsy have worse parent-reported health status ${ }^{2}$ and performance on cognitive testing compared to those without post-stroke epilepsy. ${ }^{3,4}$ However, the relationship of post-stroke epilepsy with children's neurologic function is complex and poorly understood. Our hypothesis was that children with post-stroke epilepsy have worse neurologic outcomes than those without epilepsy. We aimed to prospectively measure the effects of infarct characteristics, anti-convulsants, and epilepsy on childhood arterial ischemic stroke (AIS) outcomes.

\section{Methods.}

Seizures in Pediatric Stroke (SIPS) investigators enrolled children aged 29 days-19 years with acute AIS and identified seizures during the first year post-stroke as previously described. ${ }^{5}$ At one year, neurologists examined children to score outcomes using the Pediatric Stroke Outcome Measure (PSOM). All sites obtained ethics approval from local institutional review boards. Guardians provided written consent.

Epilepsy was defined as at least 1 unprovoked seizure occurring $>1$ month after stroke in accordance with the International League Against Epilepsy and the International Bureau for Epilepsy. ${ }^{6}$ Total PSOM scores are the sum of five subscales (right and left sensorimotor, language production, language comprehension, cognitive/behavioral) scored after neurologic examination ( 0 no deficit, 0.5 minor exam abnormality detectable with no impact on function, 1 deficit impairs function, and 2 at least one function is missing). ${ }^{7}$ A higher score indicates greater impairment. Investigators reported infarct characteristics from clinically obtained imaging.

\section{Statistics:}

PSOM scores were categorized $0-1,1.5-3,3.5-6,6.5-10$ as in prior studies. ${ }^{8}$ We used ordinal logistic regression to determine the association of predictor variables with outcome, and reported common odds ratios (OR) as a measure of effect size (the odds of a worse PSOM category in one group of children compared to another). For multivariable analysis, we included variables associated with outcome at a level of $\mathrm{P}<0.1$. 


\section{Results.}

Investigators enrolled 86 children (Figure 1). Cohort characteristics (Table 1) were similar to the previously published SIPS cohort that included neonatal AIS. ${ }^{5}$ Follow-up data were available for 80 children (4 deceased, 2 lost to follow-up) at a median of 12.1 months (IQR 11.4 - 12.9) post-stroke. At one year, $18 / 80(23 \%)$ were taking a maintenance anticonvulsant including 8/80 (10\%) who met our definition of epilepsy. No child discharged from the stroke hospitalization without anti-convulsant treatment developed epilepsy during the first year. Among 70 with PSOM scored, the median was 0.5 (IQR 0-1.5) for children without epilepsy ( $\mathrm{n}=63$ ), and 6 (IQR $0.5-10)$ for the children with epilepsy $(\mathrm{n}=7)$. The distribution of PSOM scores among children with epilepsy, children without epilepsy but treated with anti-convulsant, and those without epilepsy or anti-convulsant treatment are shown in Figure 2.

In unadjusted analyses, middle cerebral artery (MCA) territory infarct, cortical location, hemorrhagic transformation, and epilepsy at one year were each associated with poorer outcome, while disposition to home was associated with better outcome (Table 1). In our multivariable analysis, we included each of these variables and also included variables for longer duration of acute seizures, discharge from the stroke hospitalization on an anticonvulsant and anti-convulsant use at one year. In the multivariable model, only MCA stroke (OR 4.9, CI 1.1-21.3) and epilepsy (OR 24.1, CI 1.5-380) remained associated with higher PSOM scores.

\section{Discussion.}

Among pediatric AIS survivors, outcomes were worse among children who developed epilepsy during the first year post-stroke. Epilepsy was strongly associated with poorer neurologic outcome even after adjusting for infarct characteristics, anti-convulsants and other potential confounders.

The implication of a median PSOM of 0.5 in our study and a similar median one-year PSOM of 0.75 in a United Kingdom pediatric AIS cohort ${ }^{9}$ is that children's recovery after stroke is generally good. A PSOM of 0.5 indicates a deficit detectable in a single domain without functional impairment. In comparison, the subgroup of children with post-stroke epilepsy had a median PSOM of 6, indicating impaired or missing function in multiple domains.

Extensive brain injury may worsen outcomes and separately increase epilepsy risk. Infarct size has been related to neurologic outcome after childhood stroke ${ }^{8}$ and is a predictor of epilepsy after neonatal stroke. ${ }^{10} \mathrm{~A}$ second possible explanation for the association between epilepsy and neurologic outcome is that anti-convulsants could affect outcomes. While we did not find evidence for this, we observed a wide variation in anti-convulsant choice and duration of treatment that could mask an existing association. A third possibility is that epileptogenesis or the seizures that are generated could be detrimental to recovery. How a neural network in a person evolves to become seizure prone remains largely unknown. The latency period before the onset of post-stroke epilepsy provides an opportunity to study 
epileptogenesis, its affect on stroke recovery in children and the possibility of developing an intervention to improve outcome. ${ }^{11}$

The lack of measured infarct size and PedNIHSS at stroke onset to measure severity is a limitation of our study, but to account for degree of brain injury we controlled for other infarct characteristics (MCA territory, hemorrhagic transformation, cortical location) and other markers of initial stroke severity (acute seizure duration, hospital disposition). Our study is also limited by the small number of outcomes, the possibility of confounding by indication for anti-convulsant treatment and a lack of electroencephalogram data.

\section{Summary.}

Post-stroke epilepsy is associated with poorer one-year outcomes among childhood AIS survivors. While insufficient to determine causality, this association warrants further investigation.

\section{Supplementary Material}

Refer to Web version on PubMed Central for supplementary material.

\section{Funding Acknowledgements:}

Pediatric Epilepsy Research Foundation (112010-007), the NIH (2K12NS001692-11 and KL2TR000143) and the Auxilium Foundation.

\section{References.}

1. Fox CK, Glass HC, Sidney S, et al. Acute seizures predict epilepsy after childhood stroke. Ann Neurol. 2013; 74: 249-56. [PubMed: 23613472]

2. Smith SE, Vargas G, Cucchiara AJ, et al. Hemiparesis and epilepsy are associated with worse reported health status following unilateral stroke in children. Pediatr Neurol. 2015; 52: 428-34. [PubMed: 25559938]

3. De Schryver EL, Kappelle LJ, Jennekens-Schinkel A, et al. Prognosis of ischemic stroke in childhood: a long-term follow-up study. Dev Med Child Neurol. 2000; 42: 313-8. [PubMed: 10855651]

4. Studer M, Boltshauser E, Capone Mori A, et al. Factors affecting cognitive outcome in early pediatric stroke. Neurology. 2014; 82: 784-92. [PubMed: 24489131]

5. Fox CK, Mackay MT, Dowling MM, et al. Prolonged or recurrent acute seizures after pediatric arterial ischemic stroke are associated with increasing epilepsy risk. Dev Med Child Neurol. 2017; 59: 38-44. [PubMed: 27422813]

6. Fisher RS, Acevedo C, Arzimanoglou A, et al. ILAE official report: a practical clinical definition of epilepsy. Epilepsia. 2014; 55: 475-82. [PubMed: 24730690]

7. Kitchen L, Westmacott R, Friefeld S, et al. The pediatric stroke outcome measure: a validation and reliability study. Stroke; a journal of cerebral circulation. 2012; 43: 1602-8.

8. Beslow LA, Smith SE, Vossough A, et al. Hemorrhagic transformation of childhood arterial ischemic stroke. Stroke; a journal of cerebral circulation. 2011; 42: 941-6.

9. Mallick AA, Ganesan V, Kirkham FJ, et al. Outcome and recurrence one year after paediatric arterial ischaemic stroke in a population-based cohort. Ann Neurol. 2016.

10. Wusthoff CJ, Kessler SK, Vossough A, et al. Risk of later seizure after perinatal arterial ischemic stroke: a prospective cohort study. Pediatrics. 127: e1550-7. 
11. Pitkanen A, Roivainen R and Lukasiuk K. Development of epilepsy after ischaemic stroke. Lancet Neurol. 2016; 15: 185-97. [PubMed: 26597090] 


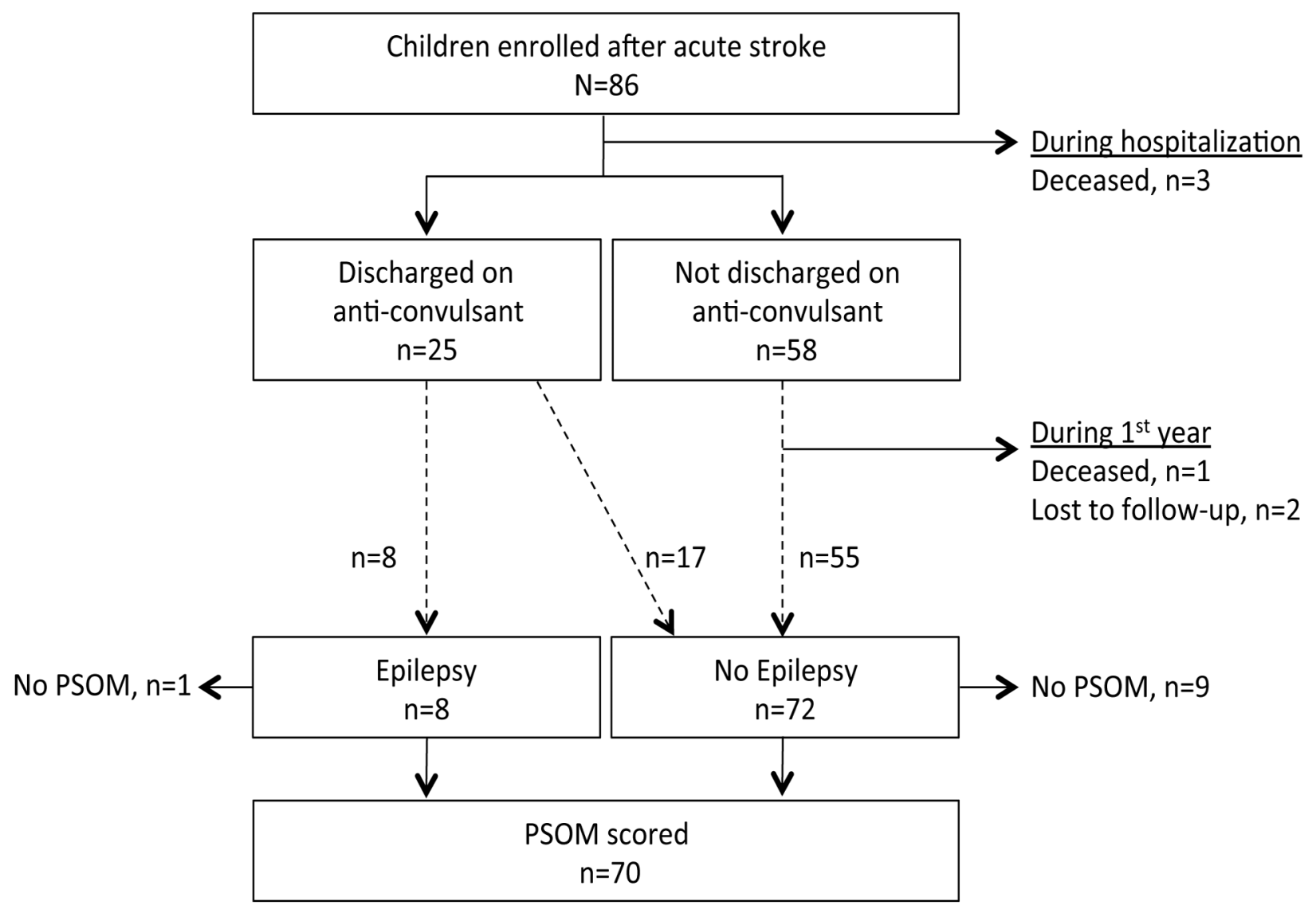

Figure 1.

Flow diagram of the childhood stroke cohort followed 1 year. 

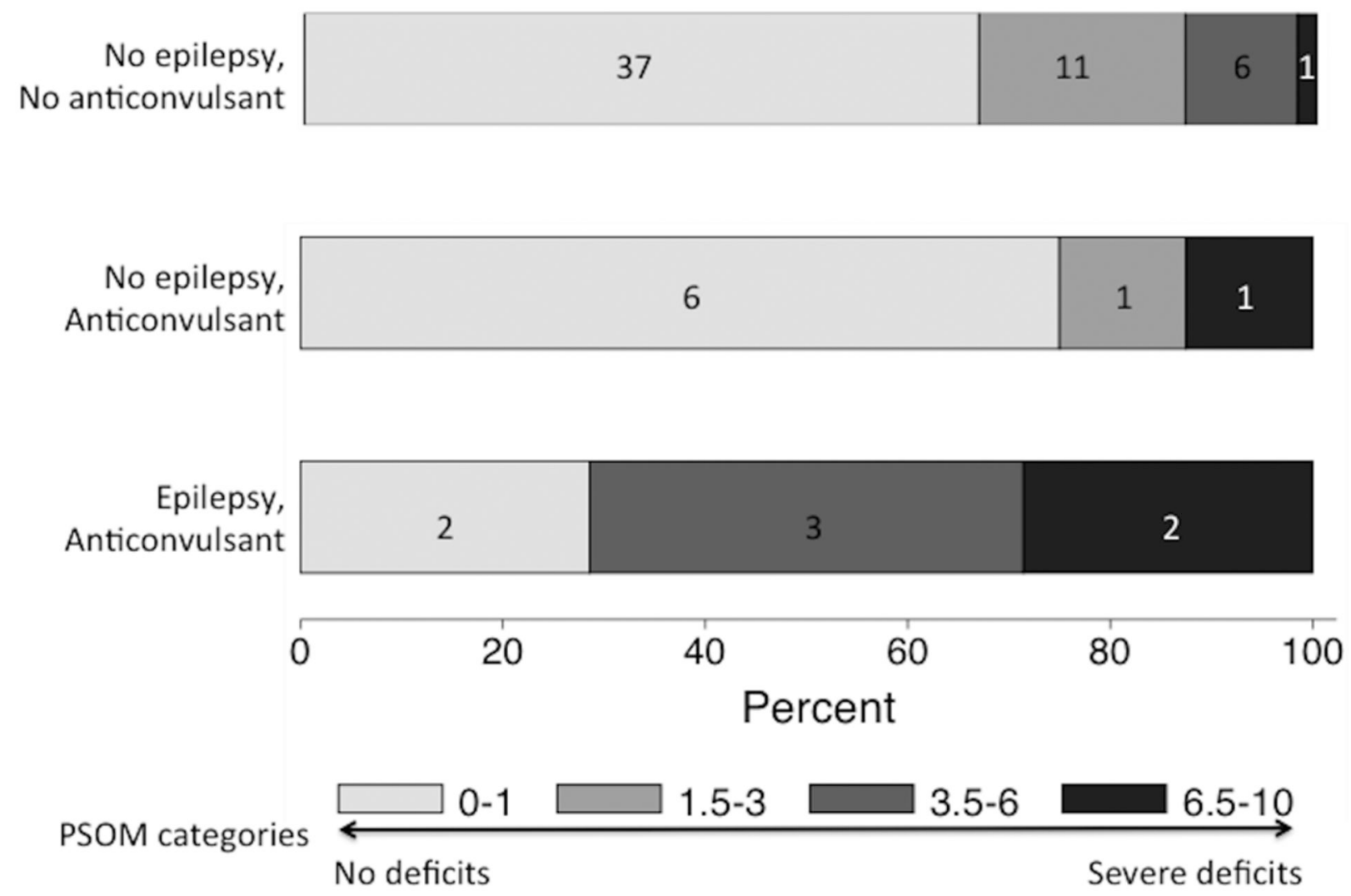

Figure 2.

Distribution of PSOM scores stratified by the presence of epilepsy and ongoing treatment with an anti-convulsant one-year post-stroke. Percent is shown on the Y-axis, with frequency counts in boxes representing each category. 
Table 1.

Factors associated with Pediatric Stroke Outcome Measurement scores one-year after arterial ischemic stroke in childhood.

\begin{tabular}{|c|c|c|c|c|c|c|c|c|}
\hline \multirow[b]{2}{*}{$N=86$} & \multirow[b]{2}{*}{$\mathbf{n}$} & \multirow[b]{2}{*}{$\%$} & \multicolumn{2}{|c|}{ Univariable } & \multicolumn{3}{|c|}{ Multivariable } & \multirow[b]{2}{*}{ P-valu } \\
\hline & & & OR & $95 \% \mathrm{CI}$ & P-value & OR & $95 \% \mathrm{CI}$ & \\
\hline \multicolumn{9}{|l|}{ Demographics } \\
\hline Male & 51 & 59 & 0.7 & $0.3-1.7$ & 0.40 & & & \\
\hline Hispanic & 15 & 17 & 1.3 & $0.4-3.9$ & 0.69 & & & \\
\hline North American & 60 & 70 & 0.8 & $0.3-2.1$ & 0.63 & & & \\
\hline Age at stroke (years; median, IQR) & 6.1 & $1.4-12.2$ & 1.0 & $0.9-1.1$ & 0.64 & & & \\
\hline \multicolumn{9}{|l|}{ Stroke risk factors } \\
\hline Arteriopathy & 33 & 38 & 1.1 & $0.4-3.0$ & 0.81 & & & \\
\hline Cardiac & 22 & 26 & 0.6 & $0.2-1.8$ & 0.34 & & & \\
\hline Acute disease & 21 & 24 & 2.0 & $0.6-6.1$ & 0.24 & & & \\
\hline Underlying chronic disease & 24 & 28 & 1.0 & $0.3-2.7$ & 0.93 & & & \\
\hline Head trauma & 7 & 8 & 2.6 & $0.5-13.1$ & 0.25 & & & \\
\hline \multicolumn{9}{|l|}{ Infarct territory and location } \\
\hline Middle cerebral artery & 53 & 62 & 4.4 & $1.3-14.7$ & 0.02 & 4.9 & $1.1-21.3$ & 0.03 \\
\hline Anterior cerebral artery & 12 & 14 & 1.6 & $0.5-5.5$ & 0.47 & & & \\
\hline Posterior cerebral artery & 18 & 21 & 0.4 & $0.1-1.6$ & 0.21 & & & \\
\hline Other vertebrobasilar & 10 & 12 & 0.2 & $0.0-1.5$ & 0.12 & & & \\
\hline Basal ganglia/thalamus & 24 & 28 & 1.1 & $0.4-3.3$ & 0.81 & & & \\
\hline Cortical infarct & 15 & 17 & 3.6 & $1.2-11.4$ & 0.03 & 2.8 & $0.7-11.3$ & 0.15 \\
\hline Multiple infarcts & 29 & 34 & 1.6 & $0.6-4.5$ & 0.40 & & & \\
\hline Hemorrhagic transformation & 3 & 3 & 10.4 & $1.1-95.9$ & 0.04 & 1.6 & $0.1-34.8$ & 0.77 \\
\hline \multicolumn{9}{|l|}{ Stroke hospitalization } \\
\hline Acute seizure ( $<7$ days post-stroke) & 32 & 37 & 2.1 & $0.8-5.7$ & 0.13 & & & \\
\hline Longest acute seizure, minutes (median*, range ${ }^{\dagger}$ ) & 0 & $0-30$ & 1.1 & $1.0-1.2$ & 0.06 & 1.0 & $0.9-1.1$ & 0.73 \\
\hline Abnormal discharge exam $t^{t} \xi$ & 50 & 60 & 2.1 & $0.7-6.4$ & 0.18 & & & \\
\hline Discharge to home ${ }^{t}$ & 70 & 84 & 0.2 & $0.1-0.8$ & 0.02 & 0.3 & $0.1-1.6$ & 0.16 \\
\hline Discharged on anti-convulsant $t^{\frac{t}{t}}$ & 25 & 30 & 2.8 & $1.0-7.8$ & 0.06 & 2.6 & $0.4-17.9$ & 0.34 \\
\hline \multicolumn{9}{|l|}{ One year follow-up $(\mathrm{N}=80)$} \\
\hline Maintenance anti-convulsant & 18 & 23 & 2.7 & $0.9-8.8$ & 0.09 & 0.4 & $0.0-4.4$ & 0.42 \\
\hline Epilepsy & 8 & 10 & 11.2 & $2.2-56.1$ & 0.00 & 24.1 & $1.5-379.7$ & 0.02 \\
\hline
\end{tabular}

